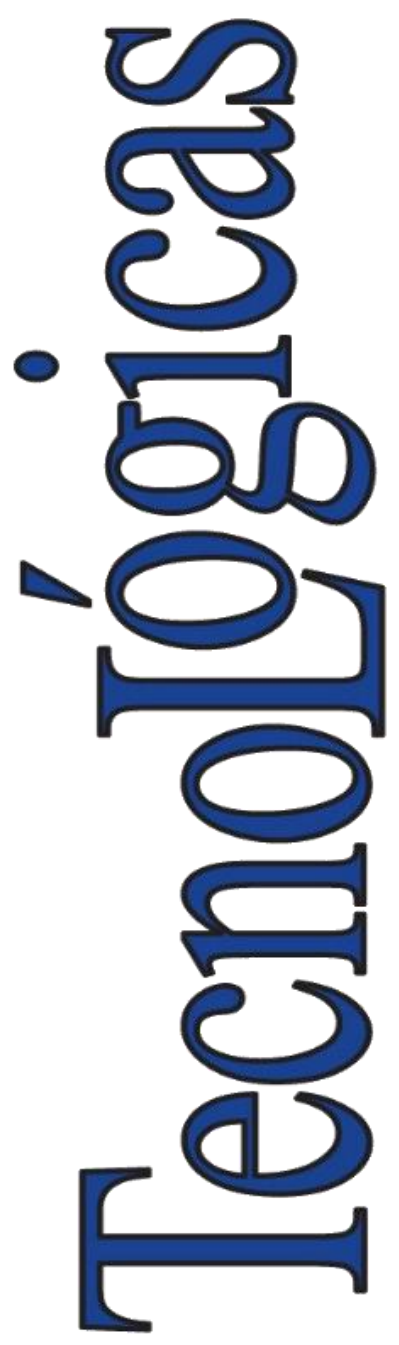

ISSN-p: 0123-7799 ISSN-e: $2256-5337$

Vol. 24, nro. 51, e1822, 2021

Recibido: 10 diciembre 2020 Aceptado: 27 mayo 2021

Disponible: 18 junio 2021

CInstituto Tecnológico Metropolitano

Este trabajo está licenciado bajo una Licencia Internacional

Creative Commons Atribución (CC BY-NC-SA)

\section{Efecto de los parámetros de operación de la extracción asistida por ultrasonido en la obtención de polifenoles de uva: una revisión}

Effect of the Operating Parameters of the UltrasoundAssisted Extraction on the Obtention of Grape Polyphenols: A Review

\author{
(D) Carolina Ramón ${ }^{1}$; \\ (D) Maritza Andrea Gil-Garzón² \\ ${ }^{1}$ Instituto Tecnológico Metropolitano, Medellín-Colombia, \\ carolinaramon239578@correo.itm.edu.co \\ 2 Instituto Tecnológico Metropolitano, Medellín-Colombia, \\ maritzagil@itm.edu.co
}

Cómo citar / How to cite

C. Ramón; M. A. Gil-Garzón, "Efecto de los parámetros de operación de la extracción asistida por ultrasonido en la obtención de polifenoles de uva: una revisión", TecnoLógicas, vol. 24, nro. 51, e1822, 2021. https://doi.org/10.22430/22565337.1822

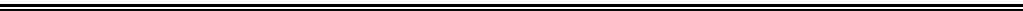




\title{
Resumen
}

Los compuestos fenólicos han sido de gran relevancia para su aplicación en industrias como la farmacéutica y la alimentaria. Esto ha llevado al estudio de métodos de extracción eficientes en cuanto a rendimiento, capacidad antioxidante y costos de operación. La extracción asistida por ultrasonido ha reportado resultados superiores en comparación con los métodos de extracción convencionales, además de ser amigable con el medio ambiente. En este sentido, este artículo tiene como objetivo evaluar el efecto de los parámetros operativos reportados con mejores resultados de extracción asistida por ultrasonido para la obtención de polifenoles en diversas variedades de uva. La revisión fue realizada en bases de datos indexadas filtrada a publicaciones en el período comprendido entre 2013 y 2020. El análisis de los resultados reportados permitió identificar los parámetros más críticos como la variación de frecuencia, potencia y tiempo, además de los rangos óptimos de operación evaluados en cada estudio que conllevaron a alcanzar un mayor rendimiento de los polifenoles totales presentes en las diferentes matrices de la uva. De igual manera, fue posible evidenciar la afectación de la temperatura sobre la concentración de los principales compuestos fenólicos característicos de la uva. Finalmente, cada transformación y resultado evaluado fue discutido a la luz de los principios físicos y químicos favorecidos por el método empleado, así como las características de termosensibilidad de los principales compuestos fenólicos como las antocianinas y flavonoles que afectan su propiedad funcional de capacidad antioxidante, de mayor interés en aplicaciones alimentarias y farmacéuticas a escala industrial.

\section{Palabras clave}

Compuestos fenólicos, extracción por ultrasonido, Vitis vinifera, capacidad antioxidante.

\begin{abstract}
Phenolic compounds have been of great relevance thanks to their application in the pharmaceutical and foods industries, among others. As a result, efficient extraction methods have been studied in terms of performance, antioxidant capacity, and operating costs. Ultrasound-assisted extraction has achieved better results than conventional extraction methods, and it is environmentally friendly. This article evaluates the effect of the operating parameters reported in the literature for ultrasound-assisted extraction that offer the best results in the obtention of polyphenols from various grape varieties. This review was carried out using indexed databases (ScienceDirect, Scopus, Scielo, PubMed, and Taylor \& Francis) filtered to publications between 2013 and 2020. The analysis of the results revealed the most critical parameters, such as the variation in frequency, power, and time, in addition to the optimal operating ranges evaluated in each study that led to a higher yield of total polyphenols present in different grape matrices. Similarly, it was found that temperature affects the concentration of the main phenolic compounds that are characteristic of grapes. Each transformation and result in the selected publications was discussed in the light of the physical and chemical principles favored by the method used in each case. Finally, this paper discusses the thermosensitivity characteristics of the main phenolic compounds in grapes; for example, anthocyanins and flavanols, which affect their antioxidant capacity and are of great interest for food and pharmaceutical applications on an industrial scale.
\end{abstract}

\section{Keywords}

Phenolics compounds, ultrasound extraction, Vitis vinifera, antioxidant capacity. 


\section{INTRODUCCIÓN}

Los compuestos polifenólicos son sustancias biológicamente activas y existen numerosas evidencias epidemiológicas, estudios in vitro, estudios en modelos animales e intervenciones en humanos, las cuales indican su beneficio al organismo, como: protección contra lesiones celulares y subcelulares, inhibición del crecimiento de tumores, activación de los sistemas de detoxificación hepáticos y bloqueo de las vías metabólicas que pueden ocasionar carcinogénesis [1]. Se ha evidenciado, además, que la promoción de hábitos saludables que eviten la generación de las especies reactivas del oxígeno, unido a la ingestión de antioxidantes como componentes fenólicos en la dieta, pueden conducir al equilibrio apropiado oxidantes/antioxidantes y consecuentemente a la prevención del cáncer [2]. Todo esto hace que el consumo de compuestos fenólicos sea de gran importancia, no sólo en el sector alimenticio sino también en el sector farmacéutico.

La diversidad de los compuestos fenólicos es tan amplia, que se pueden encontrar en una amplia gama de fuentes naturales, como las semillas y/o pericarpios de: Theobroma cacao (cacao), Campsiandra comosa Benth (chiga), Sorghum bicolor, L. Moench (sorgo), Melicoccus bijugatus (mamón). También se pueden encontrar en frutas como peras, fresas, manzanas, cerezas, duraznos, naranjas y limones; así como en hortalizas como cebollas y brócoli, y en otros alimentos como el té verde, al igual que en las uvas, manzanas, rosas, fresas y otros productos de origen vegetal, principalmente frutas y flores [3].

La uva y sus diferentes tipos de especies han sido ampliamente estudiadas como un alimento funcional debido a la presencia de antocianinas, responsables de la coloración roja de la mayoría de las frutas y vegetales, y a las cuales se les atribuye un relevante potencial antioxidante. Por otro lado, se relaciona a la uva con la "paradoja francesa", la cual indica como a pesar de que en Francia tienen una dieta rica en grasas saturadas, la incidencia de enfermedades coronarias es relativamente baja en comparación con otros países como Estados Unidos, beneficio atribuido al consumo de uvas y vinos tintos que son ricos en compuestos polifenólicos [4], [5].

La obtención de los compuestos fenólicos se ve afectada por diferentes factores dependiendo del método de extracción. Además, si se observa desde el punto de vista industrial, algunas tecnologías no resultan tan eficientes, pues representan altos consumos energéticos y tiempos prolongados, lo que se traduce en un gasto económico mayor. Por ejemplo, métodos como sólido-líquido requieren hasta veinticuatro horas de agitación [6], al igual que Soxhlet [7], que además de utilizar generalmente solventes orgánicos tóxicos, presenta tiempos de hasta veinte horas. Asimismo, estos métodos requieren separar los compuestos volátiles con un aumento de temperatura, lo que genera un riesgo de descomposición de los compuestos termosensibles. Otras tecnologías emergentes, como extracción con fluidos supercrítico [8], así como la extracción asistida con pulso [9], aún no se llevan a escala industrial en la obtención de polifenoles provenientes de la uva o su escalamiento, y en la aplicación de en otras matrices resulta en altos costos de operación [10].

Si bien la extracción asistida por ultrasonido muestra un aumento en el rendimiento de recuperación de compuestos fenólicos [11], parámetros como: la frecuencia, el tiempo, la temperatura y la relación entre solvente y muestra se deben optimizar para aumentar el rendimiento de las extracciones y no afectar las propiedades antioxidantes. Es por esto que esta revisión tiene como objetivo analizar cómo estos parámetros afectan la capacidad antioxidante y la cantidad de polifenoles totales extraídos a partir de la uva. 


\section{METOdOLOGíA}

La búsqueda de los resultados científicos reportados en el período comprendido entre 2013 y 2020 se realizó empleando cinco bases de datos indexadas (ScienceDirect, Scopus, Scielo, PubMed y Taylor \& Francis), con la ecuación de búsqueda grape AND extraction AND ultrasound AND polyphenolic. Resultado de esta ecuación, se seleccionaron las investigaciones que reportaban el contenido total de polifenoles presentes en las diferentes matrices de la uva (pulpa, cáscara, semilla y tallo) a partir de la variación en los parámetros de operación de la extracción asistida por ultrasonido. Finalmente, el análisis de los datos se realizó dividiendo las investigaciones donde variaban potencia/frecuencia, tiempo de extracción y temperatura.

\section{RESULTADOS Y DISCUSIÓN}

\subsection{Polifenoles en la uva}

Diferentes investigaciones reportan que bayas como las uvas y sus distintas variedades tienen un contenido polifenólico considerable, el cual está asociado a la capacidad antioxidante [12], [13]. Además, se conoce que el contenido fenólico de las bayas depende, no solo de la variedad de la uva, si no de otros aspectos como el clima, tipo y composición de terreno, de las prácticas de cultivo y la luminiscencia, entre otros [5]. En adición, la distribución de polifenoles varía en toda la uva, se ha reportado que la semilla es rica en flavan-3-oles, la cáscara o piel es rica en antocianinas, mientras que la pulpa presenta una baja concentración de ácidos fenólicos y sus derivados. En consecuencia, de este último hallazgo reportado, en [14] evaluaron la actividad citotóxica y antiproliferativa de un extracto purificado de cada una de las partes de la uva, donde encontraron que la actividad de la semilla fue superior en comparación a los obtenidos de la piel. Esto se puede explicar debido a la sensibilidad, durante la extracción, de las antocianinas presentes en la piel, en comparación con el aporte de los flavan-3-ol presentes en la semilla, lo que hace que su extracción sea susceptible en términos de proteger las moléculas de daños que puedan afectar su actividad antirradicalaria [14]. A continuación, se presenta en la Tabla 1 la distribución de los compuestos fenólicos en la uva [15].

Tabla 1. Distribución de los compuestos fenólicos en la uva. Fuente: elaboración propia.

\begin{tabular}{ll}
\hline Matriz & Compuestos fenólicos \\
\hline Semillas & $\begin{array}{l}\text { Flavan-3-oles: monómeros, oligómeros. } \\
\text { (OPCs) y polímeros (características las proantocianidinas). } \\
\text { Pulpa }\end{array}$ \\
Ácidos fenólicos y derivados \\
Piel & $\begin{array}{l}\text { Antocianinas, como: glicósidos oacilglicósidos (siendo la más abundante maldivina-3 } \\
\text { glucósido). }\end{array}$ \\
\hline
\end{tabular}

La extracción asistida por ultrasonido es preferida para la obtención de compuestos vegetales activos debido a su versatilidad y la capacidad de usar menos o ningún solvente orgánico, su naturaleza como método de extracción física, su simplicidad de operación, su eficiencia de extracción, su capacidad para preservar la actividad biológica de los compuestos extraídos, menos dependencia del tiempo y su capacidad para implementarse a nivel industrial, entre otros [16]. Sin embargo, es necesario ajustar los parámetros de operación para realizar la extracción de polifenoles y alcanzar un mayor rendimiento y selectividad. Entre las variables a optimizar en la extracción asistida por ultrasonido se encuentran: la 
frecuencia, la potencia, el tiempo y la temperatura que afectan directamente el rendimiento de la extracción, aunque se ha encontrado una alta dependencia del compuesto bioactivo de interés y la matriz en la cual es evaluada.

\subsection{Principio de la extracción asistida por ultrasonido}

La extracción asistida por ultrasonido es un método ampliamente utilizado para la obtención de compuestos bioactivos, debido a que es un método fácil de usar, con bajos tiempos de extracción, amigable con el medio ambiente, altos rendimientos y selectividad. El principio de este método se basa en el fenómeno de cavitación que se da por la formación, crecimiento y colapso de burbujas de vapor o gas generadas por la acción de las ondas de una frecuencia determinada [17].

En otras palabras, este fenómeno de cavitación se produce cuando se propaga una onda de presión acústica, la cual consiste en compresiones alternativas y rarefacciones en el medio de transmisión, y se lleva a cabo a lo largo de la dirección de propagación. Cuando se aplica una gran presión negativa a un líquido, las fuerzas intermoleculares de Van der Waals no son lo suficientemente fuertes como para mantener la cohesión y se forman pequeñas cavidades o microburbujas llenas de gas. Estas microburbujas no son esféricas y sufren deformaciones radiales y tangenciales antes de colapsarse. El colapso se da de manera adiabática, con un volumen tan pequeño que la energía del colapso se entrega a un número relativamente pequeño de moléculas[18], [19].

Es importante conocer la volatilidad, la tensión superficial y la viscosidad del solvente que se usará, pues estas características son cruciales para la formación de la microburbuja que se formará por el fenómeno de cavitación mencionado anteriormente. En (1) se puede observar cómo está dada la presión generada por la microburbuja:

$$
P(t)=P_{A} \sin (2 \Pi f t+\theta)
$$

Donde $\mathrm{P}_{\mathrm{A}}$ es la amplitud de presión acústica y $f$ la frecuencia de la onda de presión alterna. A medida que aumenta la frecuencia, es necesario aumentar la amplitud (o la potencia) de la irradiación para mantener la misma cantidad de energía cavitacional [20].

Todos estos fenómenos en conjunto generan una ruptura de la pared celular incrementando la permeabilidad del tejido de las plantas y facilitando la entrada del solvente al material, provocando el incremento del rendimiento del material que es extraído en un menor tiempo [21].

\subsection{Variación del rango de frecuencia y potencia}

Uno de los parámetros que se tiende a variar en las extracciones asistidas por ultrasonido para su optimización en la obtención de polifenoles presentes en la uva, es la frecuencia. Esta se ha variado en un rango que va desde $20 \mathrm{kHz}$ hasta $120 \mathrm{kHz}$. Por ejemplo, [22] en su estudio para evaluar el efecto de la frecuencia acústica, usando como solvente agua, variaron las frecuencias entre $40 \mathrm{kHz}, 80 \mathrm{kHz}$ y $120 \mathrm{kHz}$ y observaron que un aumento de la frecuencia de ultrasonido a baja potencia afectaba de manera negativa el contenido de polifenoles totales, así que finalmente concluyeron que la frecuencia óptima para la extracción de compuestos fenólicos fue a $40 \mathrm{kHz}$, con lo cual obtuvieron $32.31 \mathrm{mg}$ equivalentes de ácido gálico (GAE) / $100 \mathrm{~g}$ en peso fresco para fenoles totales. Esto se debe a que se ha observado que las bajas frecuencias generan grandes burbujas de cavitación en los disolventes de extracción que implosionan violentamente, generando un alto cizallamiento lo que asegura una mayor 
degradación celular, una mayor penetración del disolvente y una mayor tasa de extracción [23]. La relación con la potencia, como se observó anteriormente, es directamente proporcional a la frecuencia, pues al aumentar ésta la potencia debe hacerlo también. Es así como otras investigaciones han variado los parámetros de potencia frente a una misma frecuencia. En [24] variaron la potencia con una frecuencia fija de $20 \mathrm{kHz}$ en $250 \mathrm{~W}, 350 \mathrm{~W}$ y $450 \mathrm{~W}$, donde el mayor contenido de polifenoles totales fue obtenido a $450 \mathrm{~W}$, resultando con un contenido de polifenoles totales de $5.3 \mathrm{mg}$ GAE/g de muestra. En general, se ha encontrado una constante en el uso de frecuencias entre $20 \mathrm{kHz}$ a $40 \mathrm{kHz}$ y potencias entre $150 \mathrm{~W}$ a $450 \mathrm{~W}$. En [25] también reportaron que con una combinación de $40 \mathrm{kHz}$ de frecuencia con $250 \mathrm{~W}$ obtuvieron de la uva en polvo $35.12 \mathrm{mg} \mathrm{GAE} / \mathrm{g}$ de muestra. Finalmente, un alto contenido de polifenoles totales reportado usó como parámetros de extracción una frecuencia de $20 \mathrm{kHz}$ con una potencia de $150 \mathrm{~W}$ por treinta minutos, obteniendo hasta $105.81 \mathrm{mg}$ GAE/g de muestra de semilla [26].

Lo reportado conlleva a la premisa de que es importante tener una optimización de la combinación entre frecuencia y potencia para extraer una mayor cantidad de polifenoles totales. Sin embargo, es importante mencionar que usar altas frecuencias puede ser contraproducente, pues se ha reportado que genera altas cantidades de radicales libres que degradan los polifenoles y con esto reducen su actividad biológica [27]. Esto debido probablemente a la formación de burbujas inestables, las cuales colapsan y generan átomos de hidrógeno y radicales hidroxilos en soluciones acuosas que pueden propiciar la descomposición de solventes y solutos, polimerización y degradación de polifenoles [28].

En cuanto a la potencia, se ha encontrado que el aumento de esta se correlaciona directamente con el aumento de efectos sonoquímicos, es decir, producción de radicales libres, lo que promueve la degradación de los compuestos fenólicos [29].

\subsection{Variación de tiempo}

Dentro de las investigaciones reportadas se observa que el tiempo es una de las variables de mayor importancia a la hora de realizar extracciones de compuestos fenólicos. En [22], [30] evaluaron dos tiempos de extracción de compuestos fenólicos en el jugo de uva, a 15 y 30 minutos donde reportaron que lograron extraer $(30.88 \pm 2.12) \mathrm{mg}$ GAE/g de muestra en 30 minutos. También [22] concluyeron que, en un máximo de 25 minutos con condiciones fijas de $40 \mathrm{kHz}$ de frecuencia y $150 \mathrm{~W}$ de potencia, se obtiene una mayor cantidad de polifenoles totales del orujo de uva $16.65 \mathrm{mg} \mathrm{GAE} / \mathrm{g}$ de muestra. Contrastando con el ítem anterior sobre la variación de frecuencia y potencia, se puede inferir que a una menor frecuencia, estos investigadores pudieron haber obtenido un contenido de polifenoles totales en esta variación de tiempo, sin embargo, la menor frecuencia que usaron fue la que mejor resultado arrojó [22].

Por otra parte, [31] variaron los tiempos de extracción durante 1, 5, 10, 15, 20 y 30 minutos, obteniendo como resultado un máximo de compuestos fenólicos extraídos a los 5 minutos de $95.3 \mathrm{mg} \mathrm{GAE} / \mathrm{g}$ de muestra de semilla. Asimismo, demostraron que después de 5 minutos de extracción con ultrasonido, la cantidad de polifenoles totales no es significativa, e incluso, después de 10 minutos, la curva de extracción se estabiliza demostrando que la cantidad máxima extraíble ya se obtuvo. Pero es importante resaltar que esta investigación, a diferencia de las anteriormente mencionadas, fue realizada a una temperatura de $50{ }^{\circ} \mathrm{C}$, por lo tanto, es posible que después de 10 minutos de extracción, compuesto termolábiles como las antocianinas se comenzaran a degradar. En este sentido, evaluar la cinética de la extracción es sumamente importante si se pretende hacer un escalado de este proceso a nivel industrial, ya que al disminuir el tiempo de extracción se aumenta la utilidad y la eficiencia del proceso. 


\subsection{Variación de temperatura}

Este parámetro tal vez sea uno de los más críticos debido a la termosensibilidad de la mayoría de los compuestos fenólicos [32]. Sin embargo, también se sabe que la viscosidad y la tensión superficial entre solvente-muestra disminuyen de manera inversamente proporcional a la elevación de temperatura, aumentando la penetración de la matriz y con ello la tasa de extracción de polifenoles [33]. [34] usaron como temperatura de extracción $20^{\circ} \mathrm{C}, 50{ }^{\circ} \mathrm{C} \mathrm{y}$ $80{ }^{\circ} \mathrm{C}$, con tiempos de 4, 7 y 10 minutos, encontrando que la extracción óptima $23.36 \mathrm{mg}$ de GAE/g de muestra de orujo de uva, se realizó a $80^{\circ} \mathrm{C}$ con un tiempo de 4 minutos; con esta misma temperatura a 10 minutos, comenzaba a disminuir la cantidad de polifenoles totales. Por otra parte, con una temperatura de $20{ }^{\circ} \mathrm{C}$ en el mismo tiempo, la cantidad de polifenoles totales fue de $11.53 \mathrm{mg}$ de GAE/g de muestra. Lo que demuestra que, con el incremento de temperatura, disminuye la tensión superficial entre solventes y la viscosidad del mismo, haciéndolo óptimo para la extracción. También [35] informaron que el tratamiento térmico de las semillas de uva liberaba compuestos fenólicos y, por lo tanto, aumentaba la cantidad de compuestos activos. El mismo efecto podría atribuirse a la alta temperatura de extracción utilizada. Sin embargo, como se mencionó al inicio, algunos compuestos como las antocianinas que se degradan con el aumento de la temperatura, después de $40{ }^{\circ} \mathrm{C}$ las reacciones de equilibrio entre las estructuras de antocianinas son endotérmicas en la dirección de izquierda a derecha [36]: quinonoide azul $\rightleftharpoons$ flavilio rojo $\rightleftharpoons$ base de carbinol incoloro $\rightleftharpoons$ chalcona incolora, así que el calentamiento desplaza los equilibrios hacia la chalcona. En este sentido, es importante conocer las características de los compuestos bioactivos de interés para extraer y la finalidad de esta extracción, pues si la prioridad es extraer antocianinas, ya se sabe que las altas temperaturas hacen que la molécula sufra un cambio de estructura, mientras que, si la finalidad es extraer otros compuestos fenólicos y medir su capacidad antioxidante, podría ser benéfico el uso de estas temperaturas en cortos tiempos.

Se resalta, además, que se ha investigado la extracción de compuestos polifenólicos en frio, es decir, por debajo de la temperatura ambiente, donde se han logrado resultados de $10.92 \mathrm{mg}$ GAE/g de uva roja entera, a una temperatura de $10^{\circ} \mathrm{C}$ durante 6 minutos a $24 \mathrm{kHz}$ [37]. Este resultado suscita gran importancia para la industria, pues el aumento o disminución con respecto a la temperatura ambiente, incrementa los gastos de operación, al igual que con el incremento del tiempo.

\subsection{Determinación de actividad antioxidante}

Desde hace algunos años los métodos colorimétricos han sido ampliamente utilizados para determinar tanto la cantidad de polifenoles totales como la capacidad antioxidante que tienen los compuestos fenólicos y su correlación, debido a que tal vez esta sea la propiedad más importante de los compuestos polifenólicos. Dentro de los métodos más utilizados se tienen, [38], para la cuantificación de polifenoles totales, y los métodos para determinar la capacidad antioxidante, como ORAC (de sus siglas en inglés Oxygen Radical Absorbance Capacity), FRAP (Free Radical Scavenging, Ferric Reducing Antioxidant Power), DPPH, método usado para medir la estabilidad del radical 1,1-difenil-2-picrilhidrazil, y ABTS, que evalúa la estabilidad del ácido 2.2'-azino-bis(3-etilbenzotiazolin)-6-sulfónico. Sin embargo, los principios activos de estos métodos varían, como se observa en la Tabla 2 , por ende, se tiende a dificultar la comparación de estos métodos entre sí. 
Tabla 2. Métodos colorimétricos de Capacidad Antioxidante. Fuente: elaboración propia.

\begin{tabular}{|c|c|}
\hline Método & Principio de la reacción \\
\hline ORAC & $\begin{array}{l}\text { Se trata de un mecanismo HAT (hydrogen atom transfer). Mide la capacidad de captación de } \\
\text { un radical específico peroxilo, generado a partir de la molécula orgánica AAPH [39] [40]. }\end{array}$ \\
\hline FRAP & $\begin{array}{l}\text { Se trata de un mecanismo tipo SET (single electron transfer). La reducción de iones férricos } \\
\text { a ferrosos a pH bajo provoca la formación de un complejo ferroso-tripiridiltriazina coloreado. } \\
\text { Cuando un complejo de ferrictripiridiltriazina (Fe } \mathrm{Fe}^{\mathrm{III}} \mathrm{TPTZ} \text { ) se reduce a la forma ferrosa } \\
\left(\mathrm{Fe}^{\mathrm{II}}\right) \text {, se desarrolla un color azul intenso con un máximo de absorción a } 593 \mathrm{~nm}[41] \text {. }\end{array}$ \\
\hline DPPH & $\begin{array}{l}\text { Se trata de un mecanismo HAT. Mide la capacidad del agente antioxidante para ceder un } \\
\text { átomo de hidrogeno para neutralizar el radical formado por la molécula 2.2-difenil-1- } \\
\text { picrilhidrazilo, conocido por las siglas DPPH [42]. }\end{array}$ \\
\hline ABTS & $\begin{array}{l}\text { Se trata de un mecanismo tipo SET y HAT. Se generan cationes radicales de ABTS; a partir } \\
\text { de peróxido de hidrogeno } \mathrm{H}_{2} \mathrm{O}_{2} \text { u otros oxidantes fuertes como persulfato potásico en } \\
\text { presencia de metamioglobina, generan un radical intermedio ferrilmioglobina, que luego } \\
\text { reacciona con ABTS para formar el catión radical ABTS [43]. }\end{array}$ \\
\hline
\end{tabular}

Estos métodos colorimétricos son simples y con un bajo costo, lo que los hacen apropiados para aplicaciones tanto industriales como académicas. A pesar de esto, se ha generado una discusión sobre la confiabilidad de estos métodos para determinar la actividad biológica de estos compuestos; por lo tanto, los resultados obtenidos se tienden a correlacionar con otros métodos más específicos, como la cromatografía liquida. [44] comprueban la viabilidad de los métodos FRAP y ORAC comparándolos con el factor de transcripción NF- $\mathrm{kB}$, el cual se activa con la formación de especies reactivas de oxígeno (ROS). En su investigación emplean un jugo de uva y un subproducto de la vinificación evaluando el contenido de polifenoles totales por medio de Foli-Ciocalteu y HPLC y capacidad antioxidante mediante FRAP y ORAC, y la activación de NF-kB, en el cual concluyeron que la capacidad de los extractos fenólicos para inhibir la activación de NF-kB fue 36.5 \% mayor en los subproductos de la vinificación en comparación con la de jugo, lo que resultó bastante similar a los resultados de ORAC (31.1\%) y FRAP (39.5 \%). Esto comprueba la confiabilidad de los métodos colorimétricos, aunque deja abierta la discusión sobre cómo mejorar la precisión de los métodos, pues aún se encuentra cierto porcentaje de error que debe ser disminuido. Es importante también combinar estos métodos con otros más precisos para demostrar su confiabilidad, en [45] correlacionan la cantidad de fenoles extraídos con los métodos ORAC, FRAP, DPPH y ABTS, encontrando que los cuatro métodos se correlacionan altamente con la cantidad de fenoles reportada. La mayor correlación fue para el método FRAP, no obstante, se encuentra que los cuatro métodos son confiables en términos de correlación.

En la Tabla 3 se muestra un resumen de algunas investigaciones de extracciones hechas en la uva por ultrasonido, las cuales fueron publicadas entre 2015 y 2020. Se tuvo en cuenta que las investigaciones reportaran polifenoles totales y capacidad antioxidante con el fin de realizar una correlación como se observa en la Figura 1 y en la Figura 2. Esta correlación se realizó usando las investigaciones que reportaron la actividad antioxidante usando el método colorimétrico DPPH. 
Tabla 3. Investigaciones sobre extracción de compuestos fenólicos asistido por ultrasonido entre el año 2013 y 2020 que reportan polifenoles totales y capacidad antioxidante. Fuente: elaboración propia.

\begin{tabular}{|c|c|c|c|c|c|c|c|}
\hline \multirow[b]{2}{*}{ Matriz de extracción } & \multicolumn{4}{|c|}{ Parámetros de operación } & \multirow{2}{*}{$\begin{array}{l}\text { Contenido de } \\
\text { polifenoles } \\
\text { totales }\end{array}$} & \multirow{2}{*}{$\begin{array}{c}\text { Actividad } \\
\text { antioxidante }\end{array}$} & \multirow[b]{2}{*}{ Ref. } \\
\hline & $\begin{array}{c}\mathrm{f} \\
(\mathrm{kHz}) \\
\end{array}$ & $\begin{array}{c}\mathrm{t} \\
(\mathrm{min})\end{array}$ & $\begin{array}{c}\mathrm{T} \\
\left({ }^{\circ} \mathrm{C}\right)\end{array}$ & $\begin{array}{c}\mathrm{P} \\
(\mathrm{W}) \\
\end{array}$ & & & \\
\hline $\begin{array}{l}\text { Orujo de uva blanca (Vitis } \\
\text { vinifera L.) }\end{array}$ & 26 & 30 & 50 & 200 & $95.3 \mathrm{mg} \mathrm{GAE} / \mathrm{g}$ & $\begin{array}{l}375 \mathrm{mg} \text { eq a- } \\
\text { tocoferol / g }\end{array}$ & {$[31]$} \\
\hline $\begin{array}{l}\text { Semillas de uva (variedad } \\
\text { Raboso Piave) }\end{array}$ & 20 & 30 & 30 & 150 & $105.81 \mathrm{mg} \mathrm{GAE} / \mathrm{g}$ & $\begin{array}{l}109 \mathrm{mg} \text { eq a- } \\
\text { tocoferol / g }\end{array}$ & {$[26]$} \\
\hline $\begin{array}{l}\text { Orujo de uva roja (Vitis } \\
\text { vinifera L.) }\end{array}$ & 20 & 4 & 80 & 80 & $23.36 \mathrm{mg} \mathrm{GAE} / \mathrm{g}$ & $\begin{array}{l}44,78 \mathrm{mg} \mathrm{eq} \\
\mathrm{a} \text {-tocoferol/g }\end{array}$ & {$[34]$} \\
\hline $\begin{array}{l}\text { Orujo de uva roja (Vitis } \\
\text { vinifera L.) }\end{array}$ & 40 & 25 & 17 & 150 & $0.323 \mathrm{mg} \mathrm{GAE} / \mathrm{g}$ & $\begin{array}{l}0.412 \mathrm{mg} \text { eq } \\
\text { Trolox/g }\end{array}$ & {$[22]$} \\
\hline $\begin{array}{l}\text { Extracto de orujo de uva } \\
\text { roja (Vitis vinifera L.) }\end{array}$ & 25 & 60 & 20 & 300 & $438.9 \mathrm{mg} \mathrm{GAE} / \mathrm{ml}$ & $\begin{array}{l}20.95 \mathrm{mg} \mathrm{eq} \\
\text { a-tocoferol/g }\end{array}$ & {$[46]$} \\
\hline $\begin{array}{l}\text { Extracto de orujo de jugo de } \\
\text { uva } 70 \% \text { Isabel, } 15 \% \\
\text { Bordô, } 10 \% \text { Carmem and } 5 \\
\% \text { Niagara }\end{array}$ & - & 15 & 88.1 & 450 & $5.3 \mathrm{mg} \mathrm{GAE} / \mathrm{ml}$ & $\begin{array}{l}25.0 \mu \mathrm{mol} \\
\text { Trolox } / \mathrm{ml}\end{array}$ & {$[24]$} \\
\hline Orujo de uva & 20 & 3 & 20 & - & $39.5 \mathrm{mg} \mathrm{GAE} / \mathrm{g}$ & $\begin{array}{l}68,25 \mathrm{mg} \text { eq } \\
\text { Trolox/g }\end{array}$ & {$[47]$} \\
\hline Orujo de uva & 39 & 50 & 30 & 100 & $21.6 \mathrm{mg} \mathrm{GAE} / \mathrm{g}$ & $\begin{array}{l}35,8 \mathrm{mg} \text { eq } \\
\text { Trolox/g }\end{array}$ & {$[48]$} \\
\hline Tallos de Uva & 25 & 45 & 45 & 200 & $31.89 \mathrm{mg} \mathrm{GAE} / \mathrm{g}$ & $\begin{array}{l}123,9 \mu \mathrm{g} \\
\text { Trolox/ml }\end{array}$ & [49] \\
\hline Semilla de uva & 20 & 3 & 50 & 500 & $118.89 \mathrm{mg} \mathrm{GAE} / \mathrm{g}$ & $\begin{array}{l}10 \mathrm{mg} \mathrm{eq} \\
\text { Trolox/g }\end{array}$ & {$[50]$} \\
\hline Orujo de Uva & 25 & 60 & 25 & - & $4.43 \mathrm{mg}$ GAE/g & $\begin{array}{l}518 \mu \mathrm{mol} \text { eq } \\
\text { Trolox/g }\end{array}$ & {$[51]$} \\
\hline $\begin{array}{l}\text { Jugo de uva roja variedad } \\
\text { (Vitis vinifera L.) }\end{array}$ & - & 30 & 40 & - & $30.88 \mathrm{mg} \mathrm{GAE} / \mathrm{g}$ & $\begin{array}{l}2428 \mu \mathrm{mol} \mathrm{de} \\
\text { Trolox/ }\end{array}$ & {$[30]$} \\
\hline
\end{tabular}

$\mathrm{f}=$ frecuencia, $\mathrm{t}=$ tiempo, $\mathrm{T}=$ temperatura, $\mathrm{P}=$ potencia

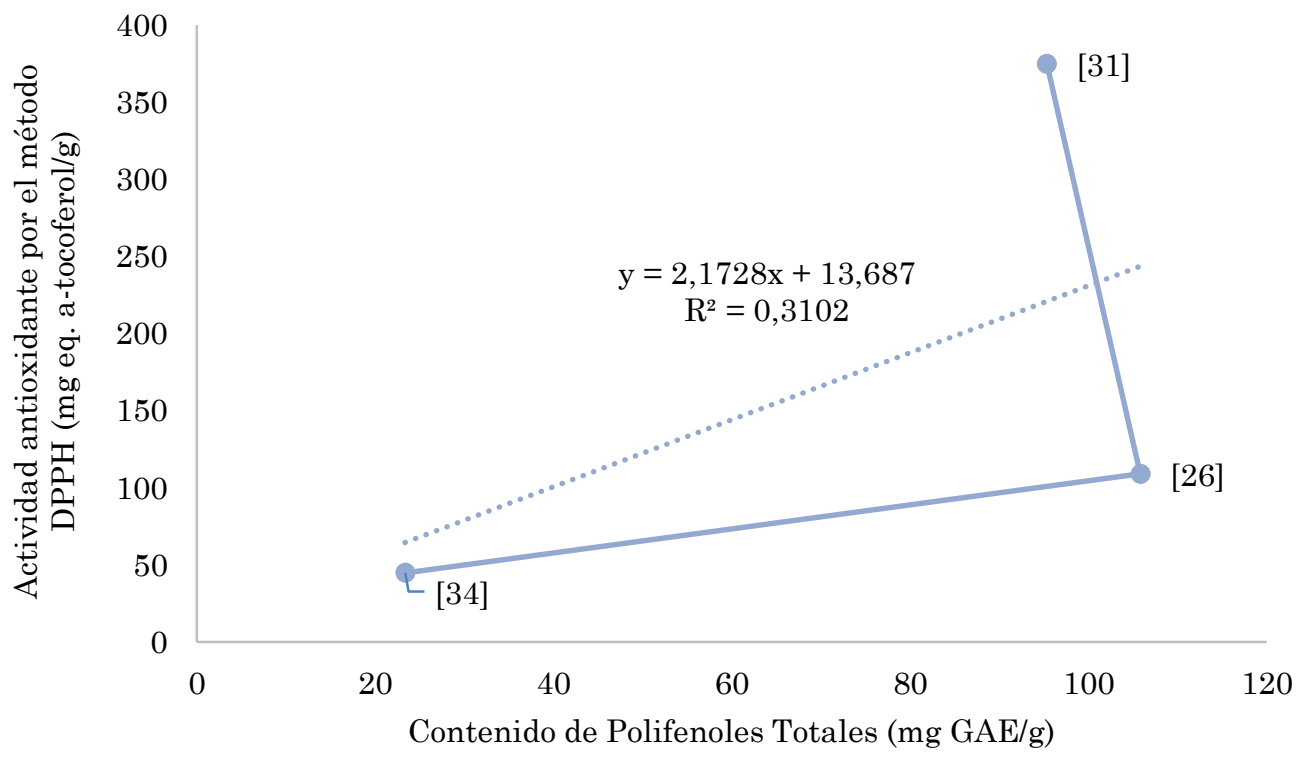

Figura 1. Polifenoles totales vs actividad antioxidante determinada por DPPH en diferentes matrices Fuente: elaboración propia. 


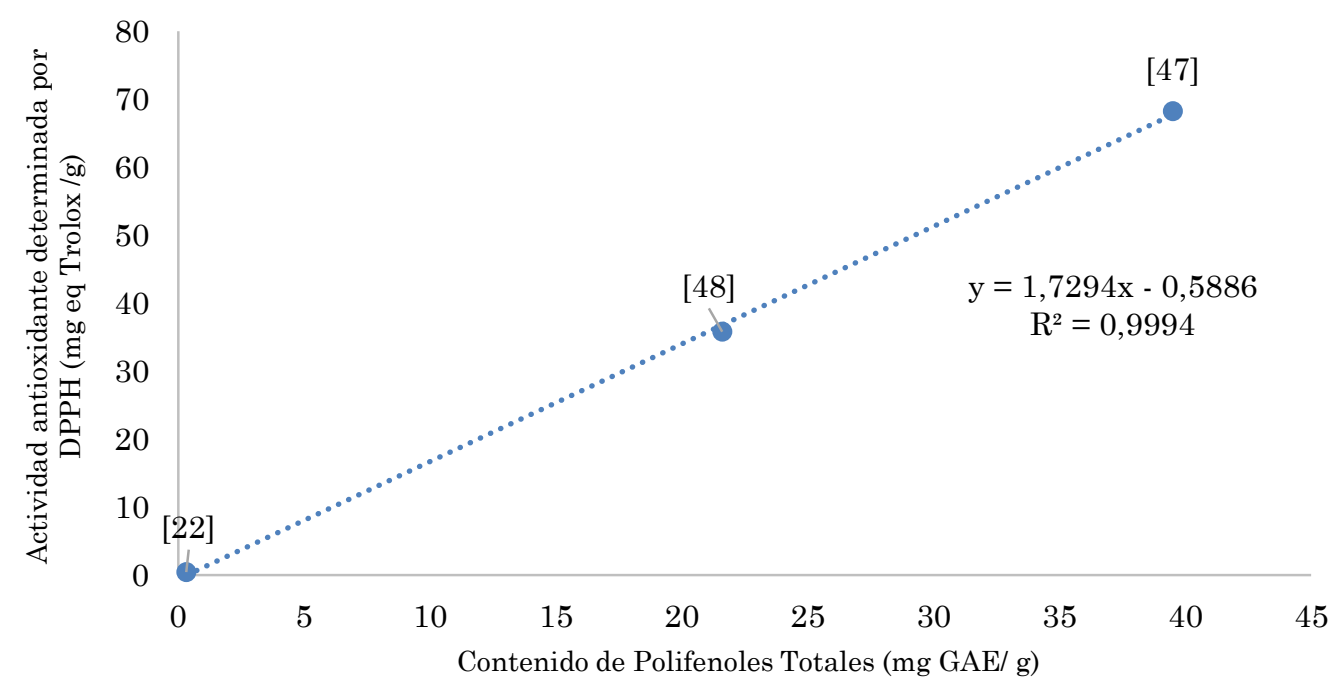

Figura 2. Polifenoles totales vs Actividad antioxidante determinada por DPPH en el Orujo de uva Fuente: elaboración propia.

Los resultados de polifenoles totales correlacionados con la actividad antioxidante varían, además de los parámetros de extracción con la matriz, pues la variedad y calidad de polifenoles totales se ven afectados según la parte de la uva que es analizada, así como su procedencia y métodos de cultivo. En la Figura 1 se puede observar cómo la correlación entre capacidad antioxidante y contenido de polifenoles totales se ve entorpecida porque las investigaciones se realizan en diferentes matrices, orujo de uva roja [34], orujo de uva blanca [31] y semillas [26]. A pesar de que en algunos casos cuentan con parámetros de operación iguales [26], [31] y tiempo de extracción de 30 minutos, la variación de los resultados se da en este caso debido a que, además de que varían matrices, también varía la frecuencia, parámetro mencionado anteriormente como clave para la extracción.

En la Figura 2 se observa que la correlación entre la cantidad de polifenoles totales y la actividad antioxidante es más lineal. Esta vez el análisis se hizo bajo la misma matriz (orujo de uva), lo que indica que hay confiabilidad en los datos reportados y que, efectivamente, la cantidad de polifenoles se relaciona directamente con la actividad antioxidante del extracto.

También es de destacar que el mayor contenido de polifenoles totales y actividad antioxidante fue la llevada a cabo a menor frecuencia [47] $20 \mathrm{kHz}$ y el menor [22] fue a $40 \mathrm{kHz}$, lo que concuerda con el análisis antes discutido sobre variación de frecuencia, pues se conoce desde la teoría que a mayor frecuencia mayor producción de radicales libres en el medio, lo cual puede ocasionar una menor cantidad de contenido de polifenoles totales al reaccionar durante el proceso con las moléculas reactivas mencionadas.

Otro aspecto importante para considerar es la relación entre solvente y la muestra. Generalmente, se prefiere el etanol, agua o mezclas de etanol/agua como solvente, debido a su polaridad acorde con las moléculas fenólicas [11].

La mezcla de etanol/agua se reporta en algunas investigaciones [31] en porciones de $57 \%$ (v/v), $53 \%$ [25] o proporciones (1:1) [11] [34]. Por otra parte, se menciona que el efecto de la proporción de disolvente a material se puede atribuir a la posibilidad de que la densidad reducida de la mezcla obtenida como resultado de una proporción más alta de disolvente a material aumente la velocidad de propagación de la onda de ultrasonido $(\boldsymbol{v})$, reduciendo el efecto de atenuación de la potencia de ultrasonido $(\boldsymbol{P})$ y aumentando la transferencia de energía $(\boldsymbol{E})$ /distancia recorrida $(\boldsymbol{d})$ por onda/tiempo $(\boldsymbol{t})$, que se puede establecer mediante (2): 


$$
P=k_{(\delta \backslash \rho)}[(E * v) / d]
$$

La eficiencia de la extracción se da debido a la eficiencia de transferencia de energía como resultado de la reducción de la densidad de la mezcla, lo que a su vez aumenta la cavitación acústica [52]. En este sentido, se encuentra que las proporcionas usadas en las investigaciones reportadas en la Tabla 2 son: 25 g/200 ml [26], 2 g/100 ml [31] y 100 g/400 ml. Teniendo en cuenta lo anterior, se pudo identificar cómo a mayor uso de solvente se mejora la eficiencia de reacción. Sin embargo, si este proceso se lleva al dominio industrial para atenuar costos, se debe encontrar una proporción adecuada donde exista un equilibrio entre solvente/costo/extracción.

\subsection{Industrialización de la tecnología de ultrasonido}

Actualmente a nivel industrial, los métodos convencionales son los preferidos para la extracción de compuestos fenólicos. Entre los estudios realizados en el escalado industrial se encuentra que se ha evaluado el impacto de la maceración asistida por ultrasonido para extraer compuestos fenólicos, en comparación a la maceración tradicional [53]. Esta investigación obtuvo como resultado que a una intensidad de $0.67 \mathrm{~W} / \mathrm{cm}^{2}, 40^{\circ} \mathrm{C}$ y 43 minutos, se logra obtener hasta un $40 \%$ de aumento en el rendimiento de extracción de polifenoles totales.

Si bien, a escala de laboratorio, en la extracción asistida por ultrasonido los rendimientos tienden a ser muy altos en comparación a los métodos tradicionales, se ha demostrado que en el escalado los rendimientos tienden a disminuir, pasando de $40 \%$ a escala de laboratorio a $15 \%$ a escala piloto [54]. Para mejorar la eficiencia se ha propuesto, además de mejorar los parámetros de operación, usar tecnologías más avanzadas, como el uso de extracciones asistidas por ultrasonido acopladas en reactores transductores múltiples, ya que se produce un aumento del flujo del solvente y este efecto ha demostrado un incremento en la cantidad de polifenoles extraíbles, resultado que han correlacionado con la actividad antioxidante conllevando a resultados óptimos [55]. Si bien esta es una solución adaptable, es importante evaluar en el campo industrial los costos correlacionados con los rendimientos obtenidos. En adición, es necesario tener en cuenta factores como diámetro del reactor y difusión del solvente en la muestra, garantizando una efectiva transferencia de masa entre solvente y soluto [56].

\section{CONCLUSIONES}

La uva es una baya que suscita gran importancia en el mundo por su consumo. Desde el siglo XX se han evaluado y correlacionado los compuestos polifenólicos con la capacidad antioxidante provenientes de la uva. Es importante tener a consideración a la hora de realizar una investigación sobre esta baya, la región, la variedad y el tipo de uva, pues la calidad y cantidad de compuestos fenólicos depende de estas variables. Por otro lado, en cuanto a los parámetros de variación del ultrasonido, se debe conocer la relación frecuencia/potencia/tiempo de extracción/temperatura para poder optimizar la extracción de los compuestos fenólicos, garantizando, por supuesto, la importante característica de la actividad antioxidante de los compuestos bioactivos. En este sentido, se encontró que, a bajas frecuencias y potencias, las extracciones reportaban mayor cantidad de polifenoles totales y mayor actividad antioxidante. En cuanto a la relación de tiempo, aunque se tienden a usar tiempos prolongados, se demostró, mediante la cinética de la reacción, que en un tiempo 
mínimo de 5 minutos se logra extraer una cantidad considerable de polifenoles totales en comparación a tiempos superiores. De igual forma, si bien las altas temperaturas ayudan a disminuir la viscosidad y disminuyen tensión superficial entre solvente/soluto, esto puede llegar a ser contraproducente si lo que se pretende extraer son compuestos termolábiles. En este sentido, si se pretende realizar la extracción de antocianinas, lo recomendable es no usar temperaturas superiores a $40{ }^{\circ} \mathrm{C}$. A nivel industrial se deben de tener en cuenta, además de los parámetros de operación del equipo, la difusión del solvente en el soluto en términos de transferencia de masa y energía de acuerdo con la forma y el diámetro del reactor a usar.

Finalmente, se demostró que, según la matriz empleada para la extracción de los compuestos fenólicos, esta influye en los resultados, tanto para polifenoles totales como para actividad antioxidante, de allí que los estudios de correlación entre actividad antioxidante de contenido de polifenoles totales, deba realizarse sobre la misma matriz.

\section{AGRADECIMIENTOS}

Los autores agradecen a la convocatoria Jóvenes Investigadores e Innovadores para grupos de investigación ITM - 2020 por su financiamiento, y al proyecto P20245 "Evaluación de la capacidad antioxidante y función biológica de un extracto y jugo obtenido a partir de la uva Isabella (Vitis labrusca) mediante la caracterización del perfil quimiométrico y de la actividad citotóxica y antitumoral: una opción para ampliar la aplicación de un ingrediente funcional" de la convocatoria interna ITM-2019 en convenio con la Corporación Universitaria Lasallista.

\section{CONFLICTOS DE INTERÉS DE LOS AUTORES}

Los autores declaramos no tener conflictos de interés ni financiero ni profesional ni personal que pueda influir de forma inapropiada en los resultados obtenidos o las interpretaciones propuestas.

\section{CONTRIBUCIÓN DE LOS AUTORES}

Tanto la concepción como la redacción y análisis de la información fue realizada de manera conjunta por los autores.

El autor de correspondencia, además, realizó una contribución sustancial al proponer la orientación de la temática y diseño metodológico de búsqueda.

Carolina Ramón-Palacio participó en la compilación de los artículos y en la revisión y análisis de estos.

Maritza Andrea-Gil Garzón participó en la definición del alcance del artículo, así como en el análisis y las conclusiones.

\section{REFERENCIAS}

[1] G. Mercado-Mercado; L. de la R. Carrillo; A. Wall-Medrano; J. A. L. Díaz; E. Álvarez-Parrilla, "Revisión Compuestos polifenólicos y capacidad antioxidante de especias típicas consumidas en México," Nutr. Hosp., vol. 28, no. 1, pp. 36-46, Jan. 2013. http://dx.doi.org/10.3305/nh.2013.28.1.6298 
[2] B. E. García Triana; A. Saldaña Bernabeu; L. Saldaña García, "El estrés oxidativo y los antioxidantes en la prevención del cáncer," Rev haban cienc méd, vol. 12, no. 2, pp. 187-196, Abr. 2013. URL

[3] F. C. Padilla; A. M. Rincón; L. Bou-Rached, "Contenido de polifenoles y actividad antioxidante de varias semillas y nueces,” Arch. Latinoam. Nutr., vol. 58, no. 3, pp. 303-308, 2008. URL

[4] G. D. González-Neves; G. C. Gatto, "Characterization of the phenolic composition and the color of Uruguayan Tannat, Cabernet Sauvignon and Merlot red wines," Inf. Tecnol., vol. 12, no. 3, pp. 9-14, 2001. $\underline{\mathrm{URL}}$

[5] M. Cañibano Alberola, "Efecto del perfil fenólico sobre las características antioxidantes de vinos tintos," (Tesis de Maestría), Escuela técnica superior de ingenierías agrarias depalencia, Universidad de Valladolid, pp. 1-30, 2012. URL

[6] L. F. Ribeiro; R. H. Ribani; T. M. G. Francisco; A. A. Soares; R. Pontarolo; C. W. I. Haminiuk, "Profile of bioactive compounds from grape pomace (Vitis vinifera and Vitis labrusca) by spectrophotometric, chromatographic and spectral analyses," J. Chromatogr. B., vol. 1007, pp. 72-80, Dec. 2015. https://doi.org/10.1016/j.jchromb.2015.11.005

[7] C. Dani et al., "Phenolic content of grapevine leaves (Vitis labrusca var. Bordo) and its neuroprotective effect against peroxide damage," Toxicol. Vitr., vol. 24, no. 1, pp. 148-153, Feb. 2010. https://doi.org/10.1016/j.tiv.2009.08.006

[8] R. Romero-Díez et al., "Microwave and ultrasound pre-treatments to enhance anthocyanins extraction from different wine lees," Food Chem., vol. 272, pp. 258-266, Jan. 2019. https://doi.org/10.1016/j.foodchem.2018.08.016

[9] M. A. Maza; J. M. Martínez; C. Delso; A. Camargo; J. Raso; I. Álvarez, "PEF-dependency on polyphenol extraction during maceration/fermentation of Grenache grapes," Innov. Food Sci. Emerg. Technol., vol. 60, Mar. 2020. https://doi.org/10.1016/j.ifset.2020.102303

[10] N. Boussetta et al., "Scale-up of high voltage electrical discharges for polyphenols extraction from grape pomace: Effect of the dynamic shock waves," Innov. Food Sci. Emerg. Technol., vol. 16, pp. 129-136, Oct. 2012. https://doi.org/10.1016/j.ifset.2012.05.004

[11] M. Bonfigli; E. Godoy; M. A. Reinheimer; N. J. Scenna, "Comparison between conventional and ultrasoundassisted techniques for extraction of anthocyanins from grape pomace. Experimental results and mathematical modeling," J. Food Eng., vol. 207, pp. 56-72, Aug. 2017. https://doi.org/10.1016/j.jfoodeng.2017.03.011

[12] G. A. Garzón, "Las antocianinas como colorantes naturales y compuestos bioactivos: revisión," Acta Biol. Colomb., vol. 13, no. 3, pp. 27-36, Dec. 2008. URL

[13] A. V. Ruales-Salcedo; A. F. Rojas-González; C. A. Cardona-Alzate, "Obtención de compuestos fenólicos a partir de residuos de uva isabella (Vitis labrusca)." Biotecnol. en el Sect. Agropecu y Agroindustrial, vol. 15, pp. 72-79, 2017. URL

[14] G. González Neves; G. Gil; F. Guzmán; M. Ferrer Baccino, "Potencial polifenólico de la uva índices propuestos y posibles aplicaciones," Comun. Sci., vol. 2, no. 2, pp. 57-69, 2011. URL

[15] J. M. Pérez-Ortiz; L. F. Alguacil; E. Salas; I. Hermosín-Gutiérrez; S. Gómez-Alonso; C. González-Martín, "Antiproliferative and cytotoxic effects of grape pomace and grape seed extracts on colorectal cancer cell lines," Food Sci. Nutr., vo. 7, no. 9, pp. 2948-2957, Sep. 2019. https://doi.org/10.1002/fsn3.1150

[16] C. Sedem Dzah et al., "The effects of ultrasound assisted extraction on yield, antioxidant, anticancer and antimicrobial activity of polyphenol extracts: A review," Food Biosci., vol. 35, Jun. 2020. https://doi.org/10.1016/j.fbio.2020.100547

[17] P. J. Arauzo; M. Lucian; L. Du; M. P. Olszewski; L. Fiori; A. Kruse, "Improving the recovery of phenolic compounds from spent coffee grounds by using hydrothermal delignification coupled with ultrasound assisted extraction," Biomass and Bioenergy, vol. 139, Aug. 2020. https://doi.org/10.1016/j.biombioe.2020.105616

[18] Y. Picó, "Ultrasound-assisted extraction for food and environmental samples," TrAC Trends Anal. Chem., vol. 43, pp. 84-99, Feb. 2013. https://doi.org/10.1016/j.trac.2012.12.005

[19] Z. Rodríguez Riera; M. Robaina Mesa; U. J. Jáuregui Haza; A. Blanco González; J. E. Rodríguez-Chanfrau, "Empleo de la radiación ultrasónica para la extracción de compuestos bioactivos provenientes de fuentes naturales. Estado actual y perspectivas," Cenic, vol. 45, pp. 139-147, 2014. URL

[20] G. Cravotto; P. Cintas, "Power ultrasound in organic synthesis: Moving cavitational chemistry from academia to innovative and large-scale applications," Chem. Soc. Rev., vol. 35, no. 2, pp. 180-196, Oct. 2005. https://doi.org/10.1039/B503848K

[21] G. V. S. Bhagya Raj; K. K. Dash, "Ultrasound-assisted extraction of phytocompounds from dragon fruit peel: Optimization, kinetics and thermodynamic studies," Ultrason. Sonochem., vol. 68, Nov. 2020. https://doi.org/10.1016/j.ultsonch.2020.105180

[22] M. Reyes González-Centeno; K. Knoerzer; H. Sabarez; S. Simal; C. Rosselló; A. Femenia, "Effect of acoustic frequency and power density on the aqueous ultrasonic-assisted extraction of grape pomace (Vitis vinifera 
L.) - A response surface approach," Ultrason. Sonochem., vol. 21, no. 6, pp. 2176-2184, Nov. 2014. https://doi.org/10.1016/j.ultsonch.2014.01.021

[23] S. Kaur Bhangu; M. Ashokkumar; J. Lee, "Ultrasound Assisted Crystallization of Paracetamol: Crystal Size Distribution and Polymorph Control," Cryst. Growth Des., vol. 16, no. 4, pp. 1934-1941, Mar. 2016. https://doi.org/10.1021/acs.cgd.5b01470

[24] C. B. Da Rocha; C. P. Zapata Noreña, "Microwave-Assisted Extraction and Ultrasound-Assisted Extraction of Bioactive Compounds from Grape Pomace," Int. J. Food Eng., vol. 16, no. 1-2, pp. 1-10, Jan. 2020. https://doi.org/10.1515/ijfe-2019-0191

[25] M. Kaleem; A. Ahmad; R. M. Amir; G. K. Raja, "Ultrasound-assisted phytochemical extraction condition optimization using response surface methodology from perlette grapes (Vitis vinifera)," Processes, vol. 7, no. 10, Oct. 2019. https://doi.org/10.3390/pr7100749

[26] C. Da Porto; E. Porretto; D. Decorti, "Comparison of ultrasound-assisted extraction with conventional extraction methods of oil and polyphenols from grape (Vitis vinifera L.) seeds," Ultrason. Sonochem., vol. 20, no. 4, pp. 1076-1080, Jul. 2013. https://doi.org/10.1016/j.ultsonch.2012.12.002

[27] C. D. Fernando; P. Soysa, "Extraction Kinetics of phytochemicals and antioxidant activity during black tea (Camellia sinensis L.) brewing," Nutr. J., vol. 14, pp. 1-7, Jul. 2015. https://doi.org/10.1186/s12937-015$\underline{0060-\mathrm{x}}$

[28] N. Masuda; A. Maruyama; T. Eguchi; T. Hirakawa; Y. Murakami, "Influence of Microbubbles on Free Radical Generation by Ultrasound in Aqueous Solution: Dependence of Ultrasound Frequency," J. Phys. Chem. B, vol. 119, no. 40, pp. 12887-12893, Sep. 2015. https://doi.org/10.1021/acs.jpcb.5b05707

[29] K. Lukić et al., "Effects of high power ultrasound treatments on the phenolic, chromatic and aroma composition of young and aged red wine," Ultrason. Sonochem., vol. 59, Dec. 2019. https://doi.org/10.1016/j.ultsonch.2019.104725

[30] M. L. Moldovan; C. Bogdan; S. Iurian; C. Roman; I. Oniga; D. Benedec, "Phenolic content and antioxidant capacity of pomace and canes extracts of some Vitis vinifera varieties cultivated in Romania," Farmacia, vol. 68, no. 1, pp. 15-21, 2020. https://doi.org/10.31925/farmacia.2020.1.3

[31] A. Natolino; C. Da Porto, "Kinetic models for conventional and ultrasound assistant extraction of polyphenols from defatted fresh and distilled grape marc and its main components skins and seeds," Chem. Eng. Res. Des., vol. 156, pp. 1-12, Apr. 2020. https://doi.org/10.1016/j.cherd.2020.01.009

[32] C. F. Timberlake, "Anthocyanins-Occurrence, extraction and chemistry," Food Chem., vol. 5, no. 1, pp. 6980, Mar. 1980. https://doi.org/10.1016/0308-8146(80)90065-5

[33] G. F. Deng; D. P. Xu; S. Li; H. Bin Li, "Optimization of ultrasound-assisted extraction of natural antioxidants from sugar apple (Annona squamosa L.) peel using response surface methodology," Molecules, vol. 20, no. 11, pp. 20448-20459, Nov. 2015. https://doi.org/10.3390/molecules201119708

[34] C. Da Porto; A. Natolino; D. Decorti, "The combined extraction of polyphenols from grape marc: Ultrasound assisted extraction followed by supercritical CO2 extraction of ultrasound-raffinate," Lwt - Food Science and Technology, vol. 61, no. 1, pp. 98-104, Apr. 2015. https://doi.org/10.1016/j.lwt.2014.11.027

[35] P. Terpinc; T Polak; N. Poklar Ulrih; H. Abramovic, "Effect of heating conditions of grape seeds on the antioxidant activity of grape seed extracts," J Agric Food Chem., vol. 59, no. 16, pp. 8639-8645, Jul. 2011. https://doi.org/10.1021/jf2016072

[36] S. Badui Dergal, Química de los alimentos, 4ta ed. Naucalpan de Juárez, México: Pearson Educación 2006.

[37] N. A. Díaz et al., "8 . Espectrofometría: Espectros de absorción y cuantificación colorimétrica de biomoléculas," Universidad de Córdoba, pp. 1-8. URL

[38] Ó. A. Muñoz-Bernal et al., "Nuevo acercamiento a la interacción del reactivo de folin-ciocalteu con azúcares durante la cuantificación de polifenoles totales," TIP, vol. 20, no. 2, pp. 23-28, Jul. 2017. https://doi.org/10.1016/j.recqb.2017.04.003

[39] J. Pérez-Jiménez, "Metodología para la Evaluación de Ingredientes Funcionales Antioxidantes. Efecto de Fibra Antioxidante de Uva en status antioxidante y parámetros de riesgo cardiovascular en humanos," (Tesis Doctoral), Departamento de Metabolismo y Nutrición, Instituto del Frío (CSIC), 2007. URL

[40] C. Zapata; P. Zapata, "Estandarización del Método ORAC como Herramienta Básica de Análisis de la Capacidad Antioxidante," Universidad CES, pp. 1-15, 2019. URL

[41] I. F. F. Benzie; J. J. Strain, "The Ferric Reducing Ability of Plasma (FRAP) as a Measure of 'Antioxidant Power": The FRAP Assay," Anal. Biochem., vol. 239, no. 1, pp. 70-76, Jul. 1996. https://doi.org/10.1006/abio.1996.0292

[42] E. Guija-Poma; M. Á. Inocente-Camones; J. Ponce-Pardo; E. Zarzosa-Norabuena, "Evaluación de la técnica 2,2-Difenil-1-Picrilhidrazilo (DPPH) para determinar capacidad antioxidante," Horiz. Médico, vol. 15, no. 1, pp. 57-60, Feb. 2015. https://doi.org/10.24265/horizmed.2015.v15n1.08

[43] C. Huet-Breña, "Métodos analíticos para la determinación de antioxidantes en muestras biológicas," (Trabajo de grado), Universidad complutense madrid, pp. 1-20, 2017. URL

[44] A. C. de Camargo et al., "Should we ban total phenolics and antioxidant screening methods? The link 
between antioxidant potential and activation of NF-kB using phenolic compounds from grape by-products," Food Chem., vol. 290, pp. 229-238, Aug. 2019. https://doi.org/10.1016/j.foodchem.2019.03.145

[45] K. Thaipong; U. Boonprakob; K. Crosby; L. Cisneros-Zevallos; D. Hawkins Byrne, "Comparison of ABTS, DPPH, FRAP, and ORAC assays for estimating antioxidant activity from guava fruit extracts," J. Food Compos. Anal., vol. 19, no. 6-7, pp. 669-675, Nov. 2006. https://doi.org/10.1016/j.jfca.2006.01.003

[46] C. Drosou; K. Kyriakopoulou; A. Bimpilas; D. Tsimogiannis; M. Krokida, "A comparative study on different extraction techniques to recover red grape pomace polyphenols from vinification byproducts," Ind. Crops Prod., vol. 75, pp. 141-149, Nov. 2015. https://doi.org/10.1016/j.indcrop.2015.05.063

[47] L. Marchante; S. Gómez Alonso; M. E. Alañón; M. S. Pérez-Coello; M. C. Díaz-Maroto, "Natural extracts from fresh and oven-dried winemaking by-products as valuable source of antioxidant compounds," Food Sci. Nutr., vol. 6, no. 6, pp. 1564-1574, 2018. https://doi.org/10.1002/fsn3.697

[48] M. González; S. Barrios; E. Budelli; N. Pérez; P. Lema; H. Heinzen, "Ultrasound assisted extraction of bioactive compounds in fresh and freeze-dried Vitis vinifera cv Tannat grape pomace," Food Bioprod. Process., vol. 124, pp. 378-386, 2020. https://doi.org/10.1016/j.fbp.2020.09.012

[49] G. Grillo et al., "Batch and flow ultrasound-assisted extraction of grape stalks: Process intensification design up to a multi-kilo scale," Antioxidants, vol. 9, no. 8, pp. 1-30, Aug. 2020. https://doi.org/10.3390/antiox9080730

[50] J. M. Poveda; L. Loarce; M. Alarcón; M. C. Díaz-Maroto; M. E. Alañón, "Revalorization of winery byproducts as source of natural preservatives obtained by means of green extraction techniques," Ind. Crops Prod., vol. 112, pp. 617-625, Feb. 2018. https://doi.org/10.1016/j.indcrop.2017.12.063

[51] V. Marinelli; L. Padalino; D. Nardiello; M. A. Del Nobile; A. Conte, "New Approach to Enrich Pasta with Polyphenols from Grape Marc," J. Chem., vol. 2015, Nov. 2015. https://doi.org/10.1155/2015/734578

[52] C. C. Coussios; C. H. Farny; G. Ter Haar; R. A. Roy, "Role of acoustic cavitation in the delivery and monitoring of cancer treatment by high-intensity focused ultrasound (HIFU)," Int. J. Hyperth., vol. 23, no. 2, pp. 105-120, Jul. 2009. https://doi.org/10.1080/02656730701194131

[53] M. T. Fernández-Ponce; B. Razi Parjikolaei; H. Nasri Lari; L. Casas; C. Mantell; E. J. Martínez de la Ossa, "Pilot-plant scale extraction of phenolic compounds from mango leaves using different green techniques: Kinetic and scale up study," Chem. Eng. J., vol. 299, pp. 420-430, Sep. 2016.

https://doi.org/10.1016/j.cej.2016.04.046

[54] A. Meullemiestre; E. Petitcolas; Z. Maache-Rezzoug; F. Chemat; S. A. Rezzoug, "Impact of ultrasound on solid-liquid extraction of phenolic compounds from maritime pine sawdust waste. Kinetics, optimization and large scale experiments," Ultrason. Sonochem., vol. 28, pp. 230-239, Jan. 2016. https://doi.org/10.1016/j.ultsonch.2015.07.022

[55] D. Pingret; A. S. Fabiano-Tixier; C. Le Bourvellec; C. M. G. C. Renard; F. Chemat, "Lab and pilot-scale ultrasound-assisted water extraction of polyphenols from apple pomace," J. Food Eng., vol. 111, no. 1, pp. 73-81, Jul. 2012. https://doi.org/10.1016/j.jfoodeng.2012.01.026

[56] L. Alexandru; G. Cravotto; L. Giordana; A. Binello; F. Chemat, "Ultrasound-assisted extraction of clove buds using batch- and flow-reactors: A comparative study on a pilot scale," Innov. Food Sci. Emerg. Technol., vol. 20, pp. 167-172, Oct. 2013. https://doi.org/10.1016/j.ifset.2013.07.011 